\title{
Evaluation of Environmental Imaging for 200kV Field Emission Cs-corrected Analytical Scanning and Transmission Electron Microscope for Multi-User Facilities
}

\author{
H. Inada, ${ }^{1}$ T. Yotsuji, ${ }^{1}$ H. Muto, ${ }^{1}$ H. Matsumoto, ${ }^{1}$ and M. Konno ${ }^{1}$.
}

1. Science \& Medical Systems Business Group, Hitachi High-Technologies Corp., Hitachinaka, Ibaraki 312-8504 Japan

In recent years the revolution in aberration correction technology has made ultrahigh resolution imaging and analysis routinely accessible on transmission electron microscope (TEM) and scanning transmission electron microscope (STEM). Particularly for catalyst or fuel cells, many scientists would like to observe the behavior and understand the degradation process in real environment (in-situ) with TEM. We have reported a evaluation of environmental TEM and STEM imaging with a newly developed analytical $200 \mathrm{kV}$ cold field emission (CFE) TEM equipped with a probe-forming aberration corrector, the model is Hitachi HF5000 (Figure 1(a)) [1][2]. The base microscope is capable of TEM, STEM imaging with bright field (BF), annular dark field (DF) detectors, and secondary electron (SE) imaging. The probe-forming aberration corrector with automated correction of up to third order aberrations allows users to obtain aberration-free STEM illumination optics with minimized effort. In addition, the stability for the newly designed high tension circuit is less than $1 \mathrm{ppm}$ which is critical to realize high energy resolution for analytical work using CFE gun and high spatial resolution for imaging. This microscope is featured high-resolution imaging with higher specimen tilt angle and sensitive X-ray analysis with dual SDD (silicon drift detector) assignment and capable of chemical analysis with a post column electron energy-loss spectrometer (EELS). For in-situ operation, we have modified a standard vacuum system suitable for high gas pressure configuration with differential pumping apertures (orifices) and additional turbomolecular pump (TMP) to improve the evacuation capability in order to keep the gun pressure low enough to operate CFE (Figure 1(b))[3]. This microscope enables to record three scanning image signal simultaneously SE, BF and DF-STEM in fast scan rate. Figure 2 shows simultaneously recorded STEM images of gold nano-particles on carbon thin film. The simultaneous observation ideally helps to reveal the processes by the surface morphology as well as motion inside of catalyst, and even SE allows atomic resolution imaging [4].

In the preliminary study, a specimen heating holder was used and increased a temperature of 200 degrees C. We employed an air as a reaction gas was induced by the gas injection nozzle that is attached on the holder. Another independent gas injection nozzle can be installed at the specimen chamber port, which permits to operate many kinds of holders.

Figure 3 shows a sequence of in-situ SE images recorded at $200 \mathrm{kV}$ to show Pt particles on the carbon surface movement and migration at a temperature of 200 degrees $\mathrm{C}$ under a pressure of $5.5 \times 10^{-3} \mathrm{~Pa}$. The vacuum pressure is measured at the main vacuum pipe. While increasing the specimen temperature we observed coalescence of some particles. Then under the air injection, some Pt particles on surface were buried in the carbon support which suggests the degradation process of the catalyst [3].

References:

[1] H Inada et al, Microsc. Microanal. 22 (2016) 32.

[2] H Inada et al, European Microsc. Congress. Vol.1 (2016) 334

[3] H Matsumoto et al, Microsc. Microanal. 27(7) (2013) 11

[4] Y Zhu et al, Nature Mater 8 (2009) 808 

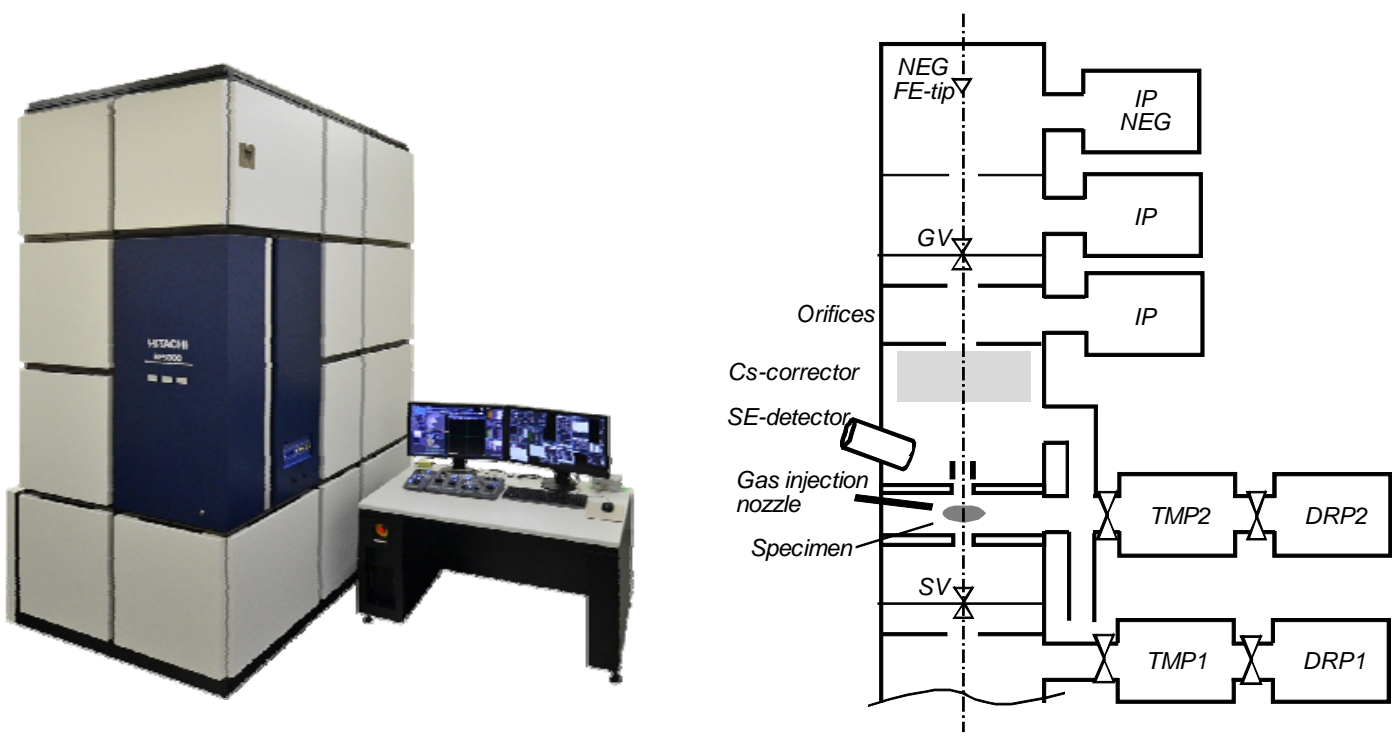

Figure 1. (a) Overview of newly designed Hitachi HF5000 200kV CFE TEM/STEM, stabilized cold field emission gun equipped analytical scanning / transmission electron microscope. And (b) a Schematic diagram of differential pumping system suitable for environmental TEM \& STEM

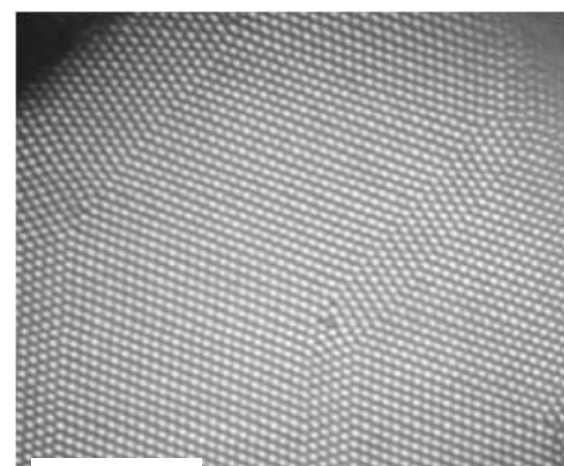

(a) DF

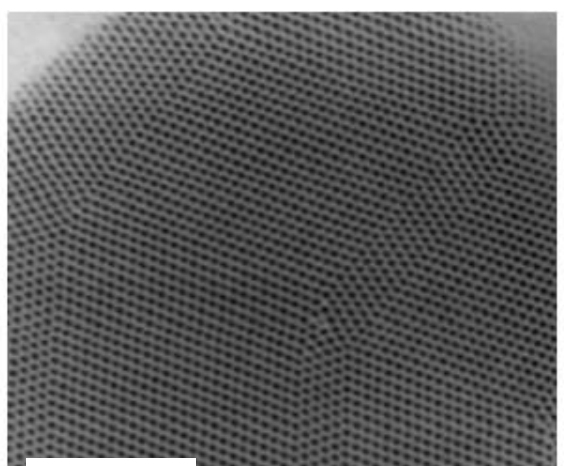

(b) BF

$5 \mathrm{~nm}$

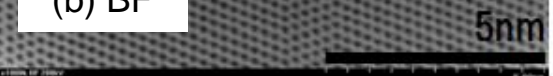

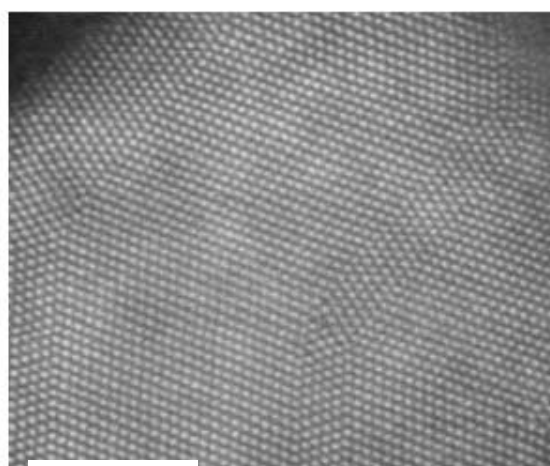

(c) SE

Figure 2. Simultaneously acquired atomic resolution STEM images of gold nano particles on carbon thin film with Hitachi HF5000 CFE TEM/STEM. (a) DF-STEM, (b) BF-STEM and (c) SE.

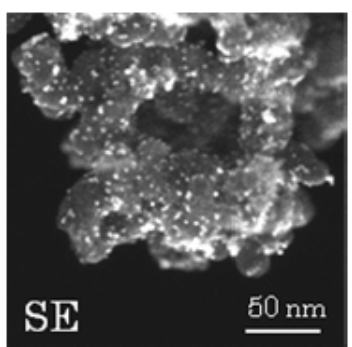

Before processing

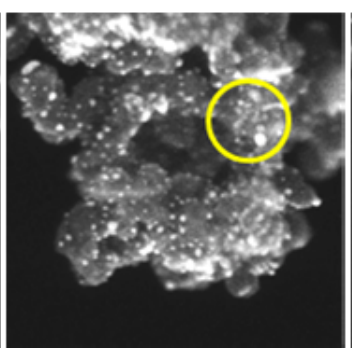

Heating 200degrees $\mathrm{C}$
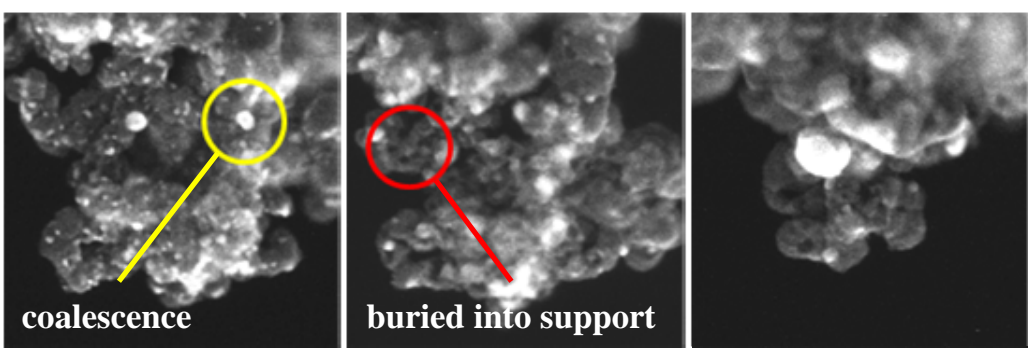

\section{air flow $(0.29 \mathrm{ccm})$}

Figure 3. Sequence of SE images showing Pt particles movement, calescence and burying into Carbon support vacuum pressure at TEM column main vacuum pipe shows $5.5 \times 10^{-3} \mathrm{~Pa}$. 